OPEN ACCESS

Edited by:

Andrea Sanson,

Università degli Studi di Padova, Italy

Reviewed by:

Angus Wilkinson,

Georgia Institute of Technology,

United States

Ranjan Mittal,

Bhabha Atomic Research Centre

India

Xianran Xing

University of Science and Technology

Beijing, China

*Correspondence:

J. Paul Attfield

j.p.attfield@ed.ac.uk

Specialty section:

This article was submitted to Physical Chemistry and Chemical

Physics,

a section of the journal

Frontiers in Chemistry

Received: 21 June 2018 Accepted: 02 August 2018

Published: 22 August 2018

Citation:

Attfield JP (2018) Mechanisms and Materials for NTE.

Front. Chem. 6:371

doi: 10.3389/fchem.2018.00371

\section{Mechanisms and Materials for NTE}

\author{
J. Paul Attfield* \\ Centre for Science at Extreme Conditions and School of Chemistry, University of Edinburgh, Edinburgh, United Kingdom
}

Negative thermal expansion (NTE) upon heating is an unusual property but is observed in many materials over varying ranges of temperature. A brief review of mechanisms for NTE and prominent materials will be presented here. Broadly there are two basic mechanisms for intrinsic NTE within a homogenous solid; structural and electronic. Structural NTE is driven by transverse vibrational motion in insulating framework-type materials e.g., $\mathrm{ZrW}_{2} \mathrm{O}_{8}$ and $\mathrm{ScF}_{3}$. Electronic NTE results from thermal changes in electronic structure or magnetism and is often associated with phase transitions. A classic example is the Invar alloy, $\mathrm{Fe}_{0.64} \mathrm{Ni}_{0.36}$, but many exotic mechanisms have been discovered more recently such as colossal NTE driven by $\mathrm{Bi}-\mathrm{Ni}$ charge transfer in the perovskite $\mathrm{BiNiO}_{3}$. In addition there are several types of NTE that result from specific sample morphologies. Several simple materials, e.g., Au, CuO, are reported to show NTE as nanoparticles but not in the bulk. Microstructural enhancements of NTE can be achieved in ceramics of materials with anisotropic thermal expansion such as beta-eucryptite and $\mathrm{Ca}_{2} \mathrm{RuO}_{4}$, and artificial NTE metamaterials can be fabricated from engineered structures of normal (positive) thermal expansion substances.

Keywords: thermal expansion, negative thermal expansion, thermal expansion coefficient, structural NTE, electronic NTE, morphological NTE

\section{INTRODUCTION}

NTE (negative thermal expansion) refers to the unusual phenomenon of volume contraction upon heating. Although most materials display positive thermal expansion (PTE) on heating, NTE is found in a wide variety of substances over varying ranges of temperature. This brief review is an attempt to summarize the mechanisms and prominent materials that show NTE. Further details may be found in more substantial reviews published by other authors in recent years (Lind, 2012; Takenaka, 2012; Chen et al., 2015; Dove and Fang, 2016; Liu et al., 2018; Mittal et al., 2018) and in the other papers in this special issue.

Control of thermal expansion is important for many applications from ceramic cooker hobs to housings for optical devices, with zero thermal expansion (ZTE) materials or composites of PTE and NTE components being particularly useful. Thermal expansion is quantified through the linear or volume (bulk) thermal expansion coefficients (TECs), $\alpha_{L}=(1 / L)(\mathrm{d} L / \mathrm{d} T)$, and $\alpha_{V}$ $=(1 / V)(\mathrm{d} V / \mathrm{d} T)$, which respectively measure the change in length $L$ or volume $V$ of an object with temperature T. $\alpha_{L}$ and $\alpha_{V}$ are typically quoted in $10^{-6} \mathrm{~K}^{-1}$ units, equivalent to ppm (parts per million) $\mathrm{K}^{-1}$ or $\mathrm{MK}^{-1}$. Isotropic substances such as simple liquids, glasses, polycrystalline ceramics, and cubic crystals, have the same $\alpha_{L}$ in all directions with $\alpha_{V}=3 \alpha_{L} . \mathrm{ZrW}_{2} \mathrm{O}_{8}$ is a famous example of a cubic NTE material (Mary et al., 1996). However uniaxial (tetragonal, hexagonal, or trigonal) crystals may have different linear TECs $\alpha_{\|}$and $\alpha_{\perp}$ parallel and perpendicular to the unique symmetry axis, respectively, and crystals with orthorhombic or lower symmetry have three different values $\alpha_{1}, \alpha_{2}$, and $\alpha_{3}$ in mutually perpendicular directions. The volume TEC is given by 
$\alpha_{V}=\alpha_{\|}+2 \alpha_{\perp}$ or $\alpha_{V}=\alpha_{1}+\alpha_{2}+\alpha_{3}$ and when the linear TECs are very different, such as a mix of negative and positive values, then highly anisotropic thermal expansion may be obtained. TECs of crystalline materials are usually measured by determining how unit cell lengths change with temperature from diffraction measurements. Direct strain gauge (dilatometry) measurements of crystals can also be used, and are particularly useful for ceramics and amorphous materials such as glasses. Diffraction and dilatometry expansion measurements can give different results due to the effects of microstructure, as discussed in section Microstructural NTE.

NTE materials have negative $\alpha_{V}$ over some temperature range. Reported $\alpha_{V}$ values vary from -1 to $-1,000 \times 10^{-6} \mathrm{~K}^{-1}$, but it is also important to consider the temperature range to which the quoted $\alpha_{V}$ refers as very large negative $\alpha_{V}$ 's may result from modest volume decreases over very narrow temperature ranges at a phase transition. For this reason it is often more useful to consider the overall volume decrease; materials with $-\Delta L / L>$ $1 \%$ and so $-\Delta V / V>3 \%$ have notably large NTE.

The thermodynamic origin of thermal expansion in solids is expressed through the relation $\alpha_{V}=\gamma C_{V} / B V$ where $C_{V}$ is heat capacity at constant $V, B=-V(\mathrm{~d} p / \mathrm{d} V)$ is the bulk modulus with $p$ being pressure, and $\gamma$ is the weighted or macroscopic Grüneisen parameter summed over values for the active phonon frequencies $\omega_{i}$ as $\gamma_{i}=-\mathrm{d}\left(\ln \omega_{i}\right) / \mathrm{d}(\ln V) . C_{V}$ and $B$ always take positive values, hence variations in the sign of $\alpha_{V}$ arise from corresponding variations in the sign of $\gamma$. NTE is often associated with other unusual lattice properties such as negative linear compressibility under applied pressure (Mittal et al., 2018) and pressure-induced softening where $\mathrm{d} B / \mathrm{d} p$ becomes negative (Dove and Fang, 2016).

Conventional PTE arises because $\gamma$ is usually positive as a consequence of the shape of the interatomic potential for bonding between two atoms, as shown in Figure 1. Anharmonicity in the shape of the potential leads to an increase in the average interatomic distance as higher vibrational states become more populated as temperature rises. As this pairwise potential shape applies qualitatively to all type of chemical bonding, it might appear that PTE should be a universal behavior, but NTE can arise from two "escapes" that circumvent the latter argument.

\section{INTRINSIC NTE MECHANISMS}

\section{Structural NTE}

The first "escape" from the universal PTE behavior expected from the interatomic potential in Figure 1 arises from the more complex vibrational properties of large arrays of atoms. Figure 2 shows some of the possible motions for a chain of atoms. Longitudinal (L) vibrations in the direction of the bonds (Figure 2A) tend to lengthen the chain as temperature increases through thermal expansion of the individual bonds via the anharmonicity of the interatomic potential (Figure 1). However, transverse $(\mathrm{T})$ motions perpendicular to the direction of the chain tend to shorten the chain-length as the amplitude of vibration increases with temperature (Figures 2B,C) and so can lead to NTE. This is sometimes known as the tension or
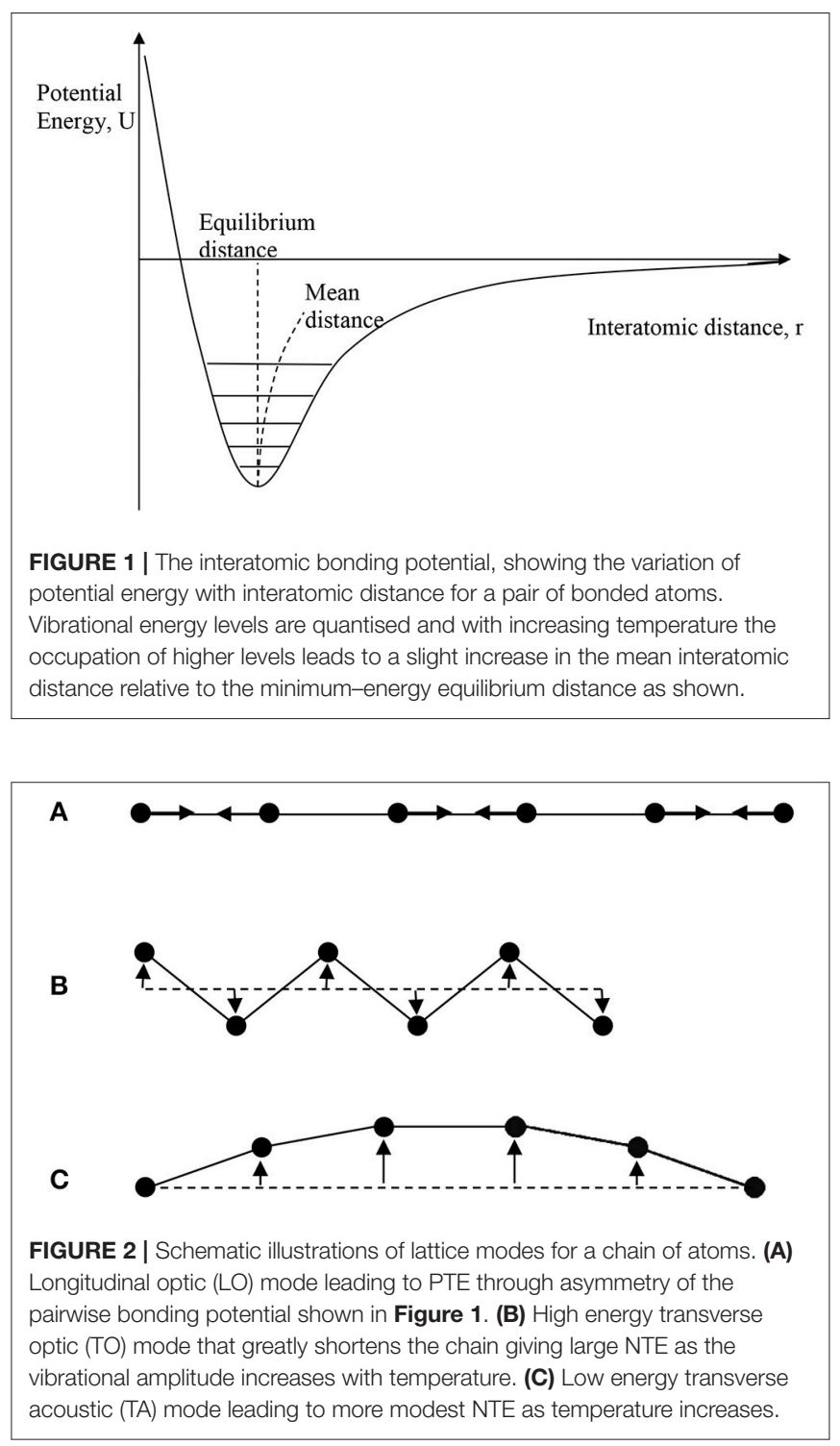

"guitar string" effect. Lattice vibrations, also known as phonons, are usefully classified as optic (O) with short wavelengths and high frequencies and energies, or acoustic (A) with long wavelengths and low frequencies and energies. Transverse optic (TO) phonons like that shown in Figure 2B lead to large chain shortening (NTE) but may only be excited at high temperatures in view of their high energies, whereas transverse acoustic (TA) modes that lead to more modest NTE are excited at lower temperatures. Detailed theoretical and experimental analyses of phonon spectra are needed to assess the contributions of TO and TA vibrations to the NTE of real materials (Dove and Fang, 2016; Mittal et al., 2018).

Structural NTE results when the shortening effects of the transverse phonon amplitudes due to bending or torsional motions outweigh the expansion effects of the longitudinal modes. Low atomic connectivity so that atoms have free space to move into during transverse motions leading to large amplitudes 
is a necessary feature for NTE to prevail. Planar 3-coordination is the maximum connectivity known to lead to negative expansion, as exemplified by graphene sheets of carbon atoms (Yoon et al., 2011). However when these sheets are stacked in the threedimensional lattice of graphite, conventional PTE arising from soft van der Waals bonding potentials in the stacking (high symmetry) direction gives a large $\alpha_{\|}=23.1 \times 10^{-6} \mathrm{~K}^{-1}$ that outweighs the NTE in the perpendicular directions $\left(\alpha_{\perp}=-0.6\right.$ $\left.\times 10^{-6} \mathrm{~K}^{-1}\right)$ leading to bulk PTE $\left(\alpha_{V}=\alpha_{\|}+2 \alpha_{\perp}=21.9 \times 10^{-6}\right.$ $\mathrm{K}^{-1}$ ) (Morgan, 1972).

Bulk structural NTE requires a large proportion of 2coordinate linker groups, connecting more highly-coordinated atoms into a three-dimensional structure. Some representative examples of structural NTE material types, with 2-connected linkers underlined, are $\mathrm{Sc}_{3} \underline{F}_{3}, \mathrm{Ag}_{2} \mathrm{O}, \mathrm{ZrW}_{2} \underline{\mathrm{O}}_{8}, \mathrm{ZrV}_{2} \underline{\mathrm{O}}_{7}, \mathrm{LiAlSi}_{4}$ (the mineral $\beta$-eucryptite), zeolitic forms of $\mathrm{SiO}_{2}$ (e.g., ITQ4) and related $\mathrm{AlPO}_{4}$ (e.g., $\left.\mathrm{AlPO}_{4}-17\right)$ frameworks, $\mathrm{Cd}(\underline{\mathrm{CN}})_{2}$, $\mathrm{Co}_{3}\left[\mathrm{Co}(\underline{\mathrm{CN}})_{6}\right]_{2}$, and metal organic frameworks such as IRMOF1 ( $\mathrm{Zn}_{4} \mathrm{O}(\underline{\mathrm{bdc}})_{3}$, where bdc is 1,4-benzodicarboxylate). These have $\alpha_{V}$ values of magnitude -20 to $-120 \times 10^{-6} \mathrm{~K}^{-1}$ over typical temperature ranges of a few hundred $\mathrm{K}$; details and citations are shown in Dove and Fang (2016). All of these materials have the majority of their atoms in the 2-connected linkers. The metal fluoride and oxide examples have 2-coordinate atoms linking tetrahedral or octahedral units together. These polyhedra tend to be rigid so the transverse vibrations of the lattice may be described in the rigid unit mode (RUM) picture (Dove and Fang, 2016).

The importance of the transverse motions of the linker atoms or groups to NTE is further demonstrated by changes observed when additional molecules or ions are inserted into NTE materials. The inserted species within cavities in the structure are adjacent to the linkers and so reduce the amplitudes of their transverse vibrations. Redox insertion of only $6 \% \mathrm{Li}$ into $\mathrm{Fe}-$ doped $\mathrm{ScF}_{3}$ switches the TEC from negative to positive (Chen et al., 2017), and the same change is found when $\mathrm{K}^{+}$or $\mathrm{H}_{2} \mathrm{O}$ is inserted into the channels of the cyanide framework material $\mathrm{YFe}(\mathrm{CN})_{6}$ (Gao et al., 2017).

\section{Electronic NTE}

A disparate group of materials, usually dense metal alloys or ceramics, display NTE that is not driven by the structural (transverse vibration) mechanism. Although they have a wide variety of physical properties, and so are sometimes described as having different NTE mechanisms, they have the common feature that NTE results from thermal changes in the interatomic bonding potential, as illustrated in Figure 3. Changes in bonding with temperature such that the interatomic potential becomes more strongly bonding can shift the curve to a smaller equilibrium distance at higher temperature. This may occur through a relatively sharp first order phase transition between two distinct states, or the potential may gradually evolve with changing temperature through a second or higher order transition. When the effect of this "escape" outweighs the usual PTE from the anharmonicity of the potentials then NTE may be observed over temperature range of the crossover.

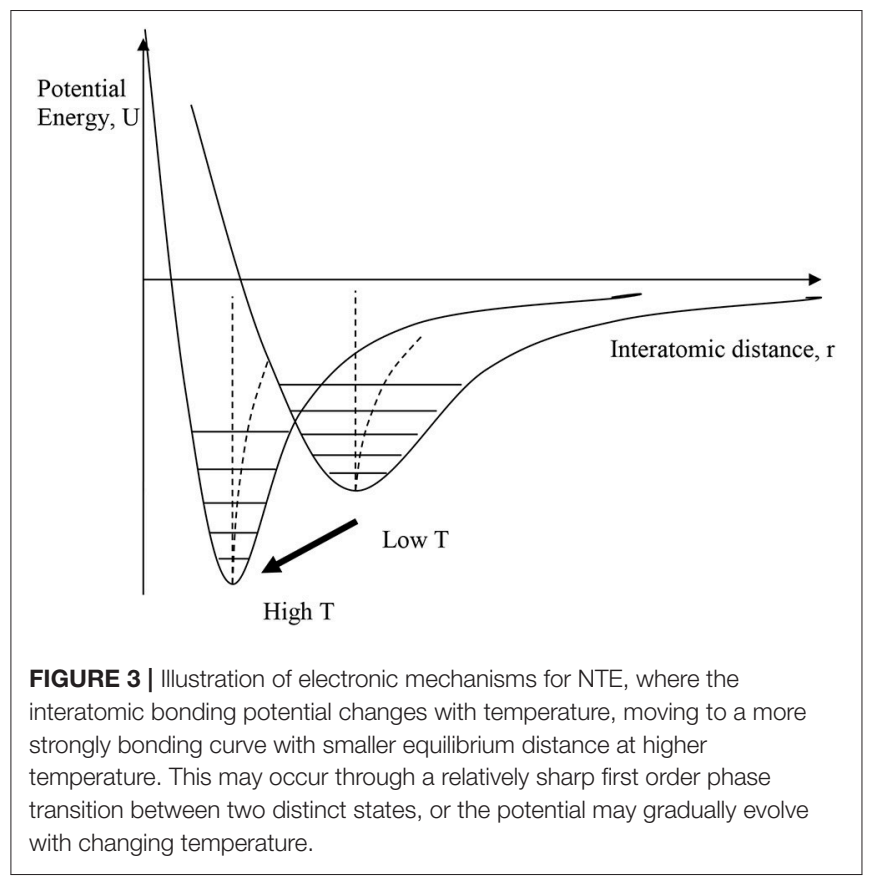

Figure 4 illustrates the schematic changes in lattice volume with temperature for a material displaying electronic NTE. Both the larger-volume low- $T$ and the smaller-volume high- $T$ states shown in Figure 3 display conventional PTE behavior, but the change between them in the crossover region with lower and upper temperatures $T_{1}$ and $T_{\mathrm{u}}$ leads to NTE. $T_{\mathrm{u}}$ often marks an ordering temperature such as a magnetic or ferroelectric Curie transition, and the lower limit $T_{1}$ is reached where the order parameter (magnetization or electric polarization) is fully saturated. In other cases such as charge transfer materials, $T_{1}$ and $T_{\mathrm{u}}$ mark the lower and upper limits of the two-phase region where the low- $T$ and high- $T$ phases coexist. The electronically-induced excess volume $\Delta V_{\text {ex }}$ relative to the extrapolated volume of the high- $T$ state, $V_{\mathrm{HT}}$, may be used to calculate the spontaneous volume striction $\Delta V_{\mathrm{ex}} / V_{\mathrm{HT}}$. Variations in the magnitude of $\Delta V_{\mathrm{ex}}$ and in the separation between $T_{1}$ and $T_{\mathrm{u}}$ may be used to tune electronic materials from reduced PTE, through ZTE to NTE behavior. A famous material that launched the study of unusual thermal expansion properties is the Invar alloy $\mathrm{Fe}_{0.64} \mathrm{Ni}_{0.36}$ named for an invariant length with a very small $\alpha_{L}=1 \times 10^{-6} \mathrm{~K}^{-1}$ (effectively ZTE) below magnetic $T_{\mathrm{C}}=500 \mathrm{~K}$.

The transitions or changes that give rise to electronic NTE are usually associated with an increase in electron delocalization on passing from the low- $T$ to the high- $T$ phase. The low- $T$ phase is more electron localized or correlated (e.g., magnetically ordered), due to electron-electron repulsions that may be described by the Hubbard $U$ energy, while the more electron delocalized or disordered high- $T$ phase is stabilized by entropy. Electrons near the Fermi level are often in $\mathrm{d}$ - or $\mathrm{f}$-states that are non-bonding or weakly antibonding, so their delocalization allows the bonding potential to become more negative and shift to shorter distance as shown in Figure 3. 


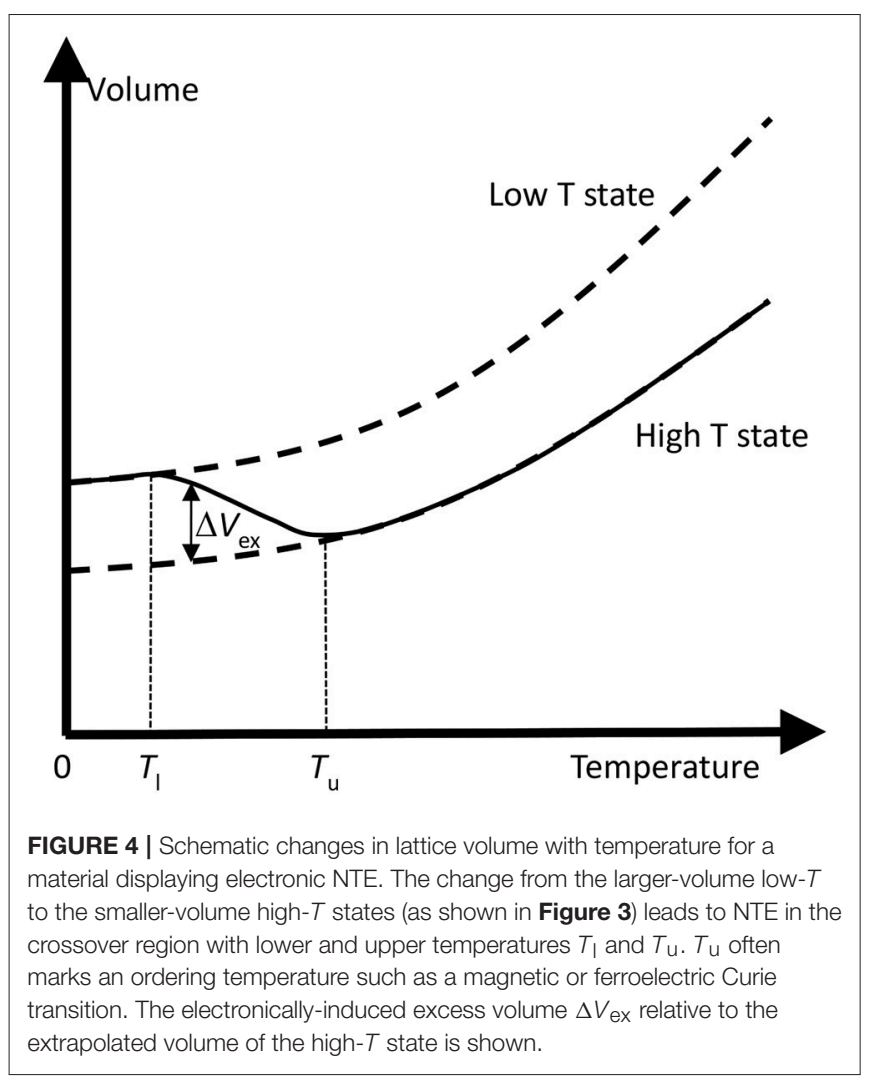

Classes of materials where electronic NTE is found are;

- Metallic magnets, notably the Invar alloy above, also $\mathrm{R}_{2} \mathrm{Fe}_{17}$ $(\mathrm{R}=$ rare earth) and related intermetallics, the permanent magnet family $\mathrm{R}_{2} \mathrm{Fe}_{14} \mathrm{~B}$, the $\mathrm{Mn}_{3}(\mathrm{Cu}, \mathrm{Ge}) \mathrm{N}$ antiperovskites, and metallic perovskite oxides $\mathrm{SrRuO}_{3}$ and manganites such as $\mathrm{La}_{0.8} \mathrm{Ba}_{0.2} \mathrm{MnO}_{3}$. NTE is usually observed over a wide temperature range below the magnetic Curie transition.

- Insulating magnets-these generally do not show NTE but some examples are found for frustrated spinels e.g., $\mathrm{CdCr}_{2} \mathrm{O}_{4}$ and $\mathrm{CdCr}_{2} \mathrm{~S}_{4}$, the multiferroic $\mathrm{Pb}\left(\mathrm{Fe}_{0.5} \mathrm{Nb}_{0.5}\right) \mathrm{O}_{3}$, and orbitally ordered $\mathrm{MnF}_{3}$, below their Curie or Néel temperatures.

- Charge transfer materials, e.g., $\mathrm{Bi}_{0.95} \mathrm{La}_{0.05} \mathrm{NiO}_{3}$, $\mathrm{LaCu}_{3} \mathrm{Fe}_{4} \mathrm{O}_{12}, \quad \mathrm{Sm}_{0.67} \mathrm{Y}_{0.33} \mathrm{~S}, \mathrm{Sm}_{2.75} \mathrm{C}_{60}$, and $\mathrm{Yb}_{8} \mathrm{Ge}_{3} \mathrm{Sb}_{5}$, can show very large NTE resulting from volume collapses at metal to insulator charge transfer transitions. $\mathrm{Bi}_{0.95} \mathrm{La}_{0.05} \mathrm{NiO}_{3}$ has $\alpha_{V}=-410 \times 10^{-6} \mathrm{~K}^{-1}$ between 300 and $370 \mathrm{~K}$, termed colossal NTE (CNTE). The oxides have intermetallic Bi/Ni or $\mathrm{Cu} / \mathrm{Fe}$ charge transfer transitions, whereas electrons released through the ionization process $\mathrm{R}^{2+} \rightarrow \mathrm{R}^{3+}+\mathrm{e}^{-}$in the $\mathrm{R}=$ $\mathrm{Sm}, \mathrm{Yb}$ materials are delocalized in the conduction band at high temperatures. NTE in $\mathrm{V}_{2} \mathrm{OPO}_{4}$ where charge ordering occurs without a metal-insulator transition was recently reported (Pachoud et al., 2018).

- Orbital ordering transitions usually associated with first-order Jahn-Teller distortions give rise to NTE in several materials. $\mathrm{Ca}_{2} \mathrm{RuO}_{4}$ has orbital order associated with a metal-insulator transition, while insulating $\mathrm{LaMnO}_{3}$ which is much studied as the parent phase of the perovskite manganites undergoes a $0.4 \%$ cell volume decrease at $750 \mathrm{~K}$ where the orbital orderdisorder transition occurs.

- Ferroelectrics associated with off-center displacements of cations to give a net polarization sometimes show NTE below their Curie transitions. Prominent examples are perovskite oxides of lead with transition metal cations showing a secondorder Jahn-Teller effect such as $\mathrm{PbTiO}_{3}, \mathrm{~Pb}\left(\mathrm{Mg}_{0.33} \mathrm{Ta}_{0.67}\right) \mathrm{O}_{3}$, and $\mathrm{Pb}\left(\mathrm{Fe}_{0.5} \mathrm{Nb}_{0.5}\right) \mathrm{O}_{3}$. Cooling below $T_{\mathrm{C}}$ leads to a change from symmetric O-M-O-M-O to asymmetric O $\cdots \cdots \mathrm{M}-$ $\mathrm{O} \cdots \cdot \mathrm{M}-\mathrm{O}$ chains of atoms leading to polarity in the chain direction. The lengthening of $\mathrm{O} \cdots \mathrm{M}$ bonds where electrons are localized in antibonding states outweighs the shortening of the M-O bonds, leading to a net expansion in the polar chain direction on cooling and hence the excess volume associated with NTE below $T_{\mathrm{C}}$. This can lead to very large volume collapses on warming, for example, $\mathrm{Pb}_{0.8} \mathrm{Bi}_{0.2} \mathrm{VO}_{3}$ shows $\Delta V / V=-7.9 \%$ around $T_{\mathrm{C}} \approx 600 \mathrm{~K}$ (Yamamoto et al., 2018).

- Superconductors sometimes show an excess volume and NTE below their critical temperatures $T_{\mathrm{c}}$, for example in $\mathrm{MgB}_{2}, \mathrm{La}_{1.85} \mathrm{Sr}_{0.15} \mathrm{CuO}_{4}$, and $\mathrm{NdFeAsO}_{0.89} \mathrm{~F}_{0.11}$. Electronphonon coupling is directly implicated in the superconducting mechanism for BCS-type $\mathrm{MgB}_{2}$, whereas the latter two materials are unconventional superconductors where a magnetic pairing mechanism may be important. Loss of bonding electron density at the Fermi level due to electron-pairing below $T_{\mathrm{c}}$ is the general cause of NTE in superconductors.

Further details and citations when not shown above are given in the comprehensive review of NTE in functional materials by Chen et al. (2015).

\section{MORPHOLOGICAL NTE}

The structural and electronic mechanisms for NTE described above apply to chemically homogenous materials such as a single crystal. However, there are further instances where NTE can arise or differ from the normal bulk behavior due to the specific morphology of the sample.

\section{Nanoparticle NTE}

A variety of materials that show bulk PTE have been found to display NTE when prepared as small particles, usually in the nanoscale regime. For example, the magnetic insulators $\mathrm{CuO}$ and $\mathrm{MnF}_{2}$ show PTE in the bulk but as $5 \mathrm{~nm}$ particles they display NTE below their Néel temperatures. CuO has a giant NTE of $\alpha_{V}$ $=-110 \times 10^{-6} \mathrm{~K}^{-1}$ between 20 and $170 \mathrm{~K}$ (Zheng et al., 2008). Nanoparticles of $\mathrm{Au}$ (Li et al., 2002) and $\mathrm{TiO}_{2}$ (Zhu et al., 2016) are also reported to display NTE.

The origin of nanoparticle NTE is usually electronic as, for example, the excess volume of $\mathrm{CuO}$ particles above follows the general behavior shown in Figure 4. Localization of ordered or correlated states tends to be enhanced at and near surfaces so the associated lattice expansion on cooling can dominate the overall behavior in small particles with a large proportion of surface atoms. The structural NTE mechanism could also play a 


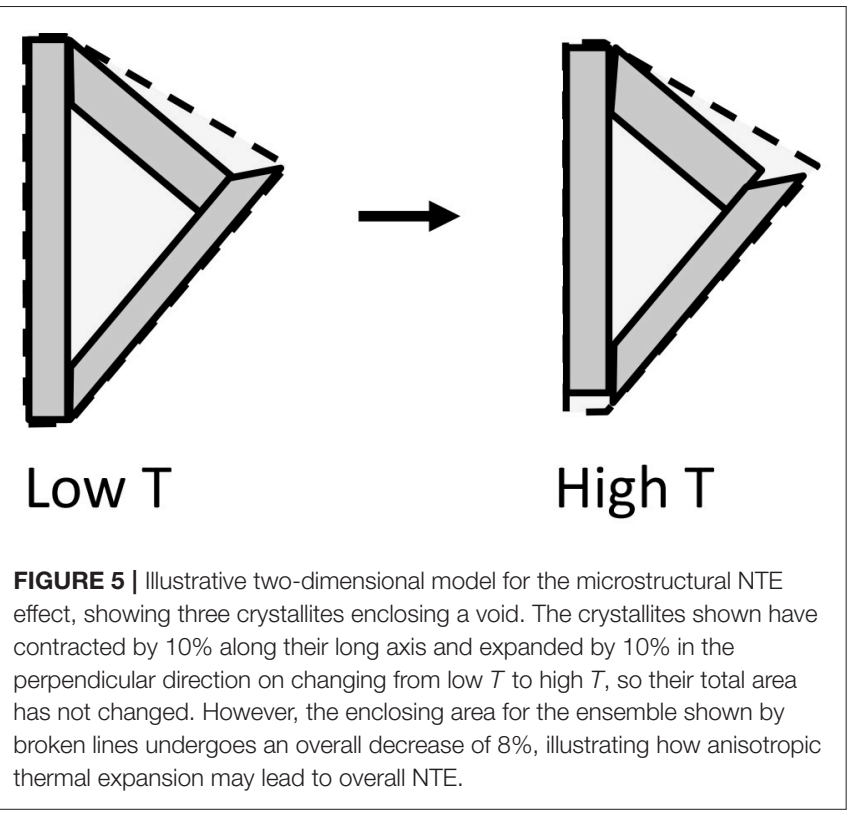

part as surface atoms have a lower connectivity than the bulk so transverse vibrational amplitudes may be enhanced.

\section{Microstructural NTE}

Direct measurement of $\alpha_{V}$ for ceramic samples sometimes gives a more negative value than that expected from crystallographic measurement of the linear TECs. The excess negative expansion is achieved by reducing the volumes of microcracks or voids within the ceramic on heating, as illustrated schematically in Figure 5. Microstructural NTE was reported in an early study of $\beta$-eucryptite $\left(\mathrm{LiAlSiO}_{4}\right)$ (Gillery and Bush, 1959) and very large effects have recently been discovered in $\mathrm{Ca}_{2} \mathrm{RuO}_{4}$ (Takenaka et al., 2017a). These ceramic materials both have anisotropic thermal expansion with one strongly negative coefficient that dominates the expansion of their ceramics. For example, on heating from 150 to $340 \mathrm{~K}$, the orthorhombic $a, b$, and $c$ axes of $\mathrm{Ca}_{2} \mathrm{RuO}_{4}$ show length changes of $-0.6,-5.0$, and $+4.5 \%$ respectively, so an overall $\Delta V / V=-1.1 \%$ is expected. However an $80 \%$ dense ceramic sample gave $\Delta V / V=-6.7 \%$ revealing a substantial microstructural NTE effect.

Anisotropic thermal expansion leads to stresses at grain boundaries that result in microcracking in $\beta$-eucryptite ceramics on cooling to room temperature after sintering. Reduction of the microcrack volumes on subsequent heating results in bulk NTE and an enhancement of the excess NTE with increasing grain size (and hence internal microcrack volume) has been established (Pelletant et al., 2012). However, this is less problematic for $\mathrm{Ca}_{2} \mathrm{RuO}_{4}$-based ceramics, which have been combined with epoxy resin to generate ZTE materials that are stable to microcracking on thermal cycling (Takenaka et al., 2017b).

\section{Metamaterials NTE}

Artificial structures consisting of two or more materials with different TECs (which may all be positive) can be engineered

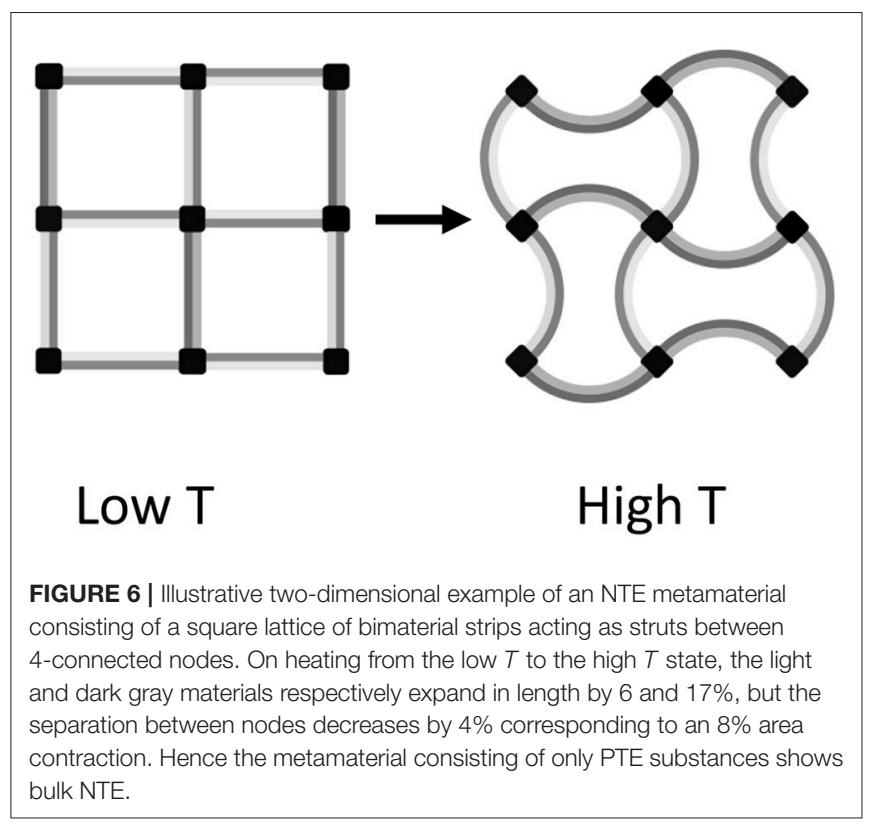

to contract when heating. A simple example based on bonded strips of two materials with different positive TECs is shown in Figure 6. The structure contracts on heating through reduction of the volume of internal voids, which outweighs the increase in volume of the materials themselves. Designs for threedimensional cellular metamaterials with varying TECs based on the use of such bimaterial connectors were proposed by Lakes (2007).

Recent developments in additive manufacturing through three-dimensional printing technologies have enabled metamaterials with bulk NTE to be generated. In a recent example, a two-component polymer metamaterial showed bulk negative expansion with $\alpha_{L}=-50 \times 10^{-6} \mathrm{~K}^{-1}$ although the individual components had PTE with an average $\alpha_{L}=40 \times 10^{-6}$ $\mathrm{K}^{-1}$ (Qu et al., 2017).

\section{SUMMARY}

Although many diverse examples of NTE materials are reported, they may be classified according to two types of intrinsic mechanism. Electronic NTE arises from reduction in first-neighbor distances upon heating due to changes in the interatomic potential. These changes may arise from a variety of physical property transitions such as magnetism, charge transfer, ferroelectricity, and superconductivity that alter the distribution of electron density. Structural NTE occurs through reduction in second- or higher- neighbor distances upon heating due to dominant effects of transverse vibrations such as bending or torsional modes, and is found in many framework-type materials with a high proportion of 2 -connected linkers.

The intrinsic NTE mechanisms may be enhanced in nanoparticles, most likely through electronic effects from localization of ordered or correlated states near surfaces. Further 
morphological mechanisms for NTE of bulk artifacts result from reducing the volume of internal voids upon heating. Microstructural NTE is found for ceramics of materials having anisotropic thermal expansion coefficients. Artificial structures of substances with different expansion coefficients may be used to generate metamaterials with NTE even when the individual components have PTE behavior.

\section{AUTHOR CONTRIBUTIONS}

The author confirms being the sole contributor of this work and approved it for publication.

\section{REFERENCES}

Chen, J., Gao, Q., Sanson, A., Jiang, X., Huang, Q., Carnera, A., et al. (2017). Tunable thermal expansion in framework materials through redox intercalation. Nat. Commun. 8:14441. doi: 10.1038/ncomms14441

Chen, J., Hu, L., Deng, J., and Xing, X. (2015). Negative thermal expansion in functional materials: controllable thermal expansion by chemical modifications. Chem. Soc. Rev. 44, 3522-3567. doi: 10.1039/C4CS00461B

Dove, M. T., and Fang, H. (2016). Negative thermal expansion and associated anomalous physical properties: review of the lattice dynamics theoretical foundation. Rep. Prog. Phys. 79:066503. doi: 10.1088/0034-4885/79/6/066503

Gao, Q.,Chen, J., Sun, Q., Chang, D., Huang, Q., Wu, H., et al. (2017). Switching between giant positive and negative thermal expansions of a $\mathrm{YFe}(\mathrm{CN}) 6$-based Prussian blue analogue induced by guest species. Angew. Chem. Int. Ed. 56, 9023-9028. doi: 10.1002/anie.201702955

Gillery, F. H., and Bush, E. A. (1959). Thermal contraction of $\beta$-eucryptite-. (Li2O.Al2O3.2SiO2) by X-ray and dilatometer methods. J. Am. Cer. Soc. 42, 175-177. doi: 10.1111/j.1151-2916.1959.tb12942.x

Lakes, R. (2007). Cellular solids with tunable positive or negative thermal expansion of unbounded magnitude. Appl. Phys. Lett. 90:221905. doi: $10.1063 / 1.2743951$

Li, W. H., Wu, S. Y., Yang, C. C., Lai, S. K., Lee, K. C., Huang, H. L., et al. (2002). Thermal contraction of Au nanoparticles. Phys. Rev. Lett. 89:135504. doi: 10.1103/PhysRevLett.89.135504

Lind, C. (2012). Two decades of negative thermal expansion research: where do we stand? Materials 5, 1125-1154. doi: 10.3390/ma5061125

Liu, Z., Gao, Q., Chen, J., Deng, J., Lin, K., and Xing, X. R. (2018). Negative thermal expansion in molecular materials. Chem. Commun. 54, 5164-5176. doi: 10.1039/C8CC03219J

Mary, T. A., Evans, J. S. O., Vogt, T., and Sleight, A. W. (1996). Negative thermal expansion from 0.3 to 1050 Kelvin in ZrW2O8. Science 272, 90-92. doi: $10.1126 /$ science. 272.5258 .90

Mittal, R., Gupta, M. K., and Chaplot, S. L. (2018). Phonons and anomalous thermal expansion behavior in crystalline solids. Prog. Materials Sci. 92, 360-445. doi: 10.1016/j.pmatsci.2017.10.002

Morgan, W. C. (1972). Thermal expansion coefficients of graphite crystals. Carbon 10, 73-79. doi: 10.1016/0008-6223(72)90011-5

Pachoud, E., Cumby, J., Lithgow, C. T., and Attfield, J. P. (2018). Charge order and negative thermal expansion in V2OPO4. J. Am. Chem. Soc. 140, 636-641. doi: $10.1021 /$ jacs.7b09441

\section{FUNDING}

The author acknowledges financial support from EPSRC.

\section{ACKNOWLEDGMENTS}

This paper is based upon a talk presented at the 2nd International Symposium on Negative Thermal Expansion and Related Materials (ISNTE-II) at Tokyo Institute of Technology, Yokohama, Japan in December 2017. The author thanks the organizers and participants for their contributions that helped to shape the ideas presented in this paper.

Pelletant, A., Reveron, H., Chêvalier, J., Fantozzi, G., Blanchard, L., Guinot, F., et al. (2012). Grain size dependence of pure $\beta$-eucryptite thermal expansion coefficient. Mater. Lett. 66, 68-71. doi: 10.1016/j.matlet.2011. 07.107

Qu, J., Kadic, M., Naber, A., and Wegener, M. (2017). Micro-structured twocomponent 3D metamaterials with negative thermal-expansion coefficient from positive constituents. Sci. Rep. 7:40643. doi: 10.1038/srep40643

Takenaka, K. (2012). Negative thermal expansion materials: technological key for control of thermal expansion. Sci. Technol. Adv. Mater. 13:013001. doi: 10.1088/1468-6996/13/1/013001

Takenaka, K., Okamoto, Y., Shinoda, T., Katayama, N., and Sakai, Y. (2017a). Colossal negative thermal expansion in reduced layered ruthenate. Nature Comm. 8:14102. doi: 10.1038/ncomms 14102

Takenaka, K., Shinoda, T., Inoue, N., Okamoto, Y., Katayama, N., and Sakai, Y., et al. (2017b). Giant negative thermal expansion in Fe-doped layered ruthenate ceramics. Appl. Phys. Express 10:115501. doi: 10.7567/APEX.10.1 15501

Yamamoto, H., Imai, T., Sakai, Y., and Azuma, M. (2018). Colossal negative thermal expansion in electron-doped $\mathrm{PbVO} 3$ perovskites. Angew. Chem. Int. Ed. 57, 1-5. doi: 10.1002/anie.201804082

Yoon, D., Son, Y. W., and Cheong, H. (2011). Negative thermal expansion coefficient of graphene measured by Raman spectroscopy. Nano Lett. 11, 3227-3231. doi: 10.1021/nl201488g

Zheng, X. G., Kubozono, H., Yamada, H., Kato, K., Ishiwata, Y., and Xu, C. N. (2008). Giant negative thermal expansion in magnetic nanocrystals. Nature Nat. Nanotechnol. 3, 724-726. doi: 10.1038/nnano.2008.309

Zhu, H., Li, Q., Ren, Y., Fan, L., Chen, J., Deng, J., et al. (2016). Hydration and thermal expansion in anatase nanoparticles. Adv Mater. 28, 6894-6899. doi: 10.1002/adma.201600973

Conflict of Interest Statement: The author declares that the research was conducted in the absence of any commercial or financial relationships that could be construed as a potential conflict of interest.

Copyright (C) 2018 Attfield. This is an open-access article distributed under the terms of the Creative Commons Attribution License (CC BY). The use, distribution or reproduction in other forums is permitted, provided the original author(s) and the copyright owner(s) are credited and that the original publication in this journal is cited, in accordance with accepted academic practice. No use, distribution or reproduction is permitted which does not comply with these terms. 